\title{
Comment on "Uniformization of the transverse beam profile by means of nonlinear focusing method"
}

\author{
Robert E. Shafer \\ 1322 Big Rock Loop, Los Alamos, New Mexico 87544, USA \\ (Received 5 November 2007; published 20 March 2008)
}

\begin{abstract}
This Comment presents unpublished and published work done by Los Alamos National Laboratory (LANL) in the period 1990 to 1999 using the nonlinear focusing method outlined in the recent publication by Yuri et al. [Phys. Rev. ST Accel. Beams 10, 104001 (2007)]. The LANL work included theory, design, modeling, and testing of nonlinear focusing beam "expanders" for use with high energy proton beams, including the proposed 1.7-GeV, 100-mA cw proton Accelerator for the Production of Tritium (APT). For several reasons listed in the text, the APT nonlinear focusing beam expander design was replaced with a high-frequency beam raster system.
\end{abstract}

DOI: 10.1103/PhysRevSTAB.11.039001

PACS numbers: 41.75.-i, 41.85.Ew, 29.27.Eg

Los Alamos National Laboratory (LANL) has been involved in the theory, design, modeling, and testing of beam expanders using the nonlinear focusing method since 1990 [1-3]. The Los Alamos Accelerator for Production of Tritium (APT) nonlinear beam expander was designed to uniformly illuminate a $17 \mathrm{~cm}$ wide by $170 \mathrm{~cm}$ high target area using a $1.7-\mathrm{GeV}, 100-$ milliamp $\mathrm{cw}$ proton beam. Because of the very high average beam power (170 MW), even very small beam losses could lead to very high activation losses that would damage magnet coil insulation and make remote handling necessary for beam line maintenance. For this reason, the beam losses in the accelerator and beam expander were modeled extensively using particle-in-cell codes for space-charge dominated beams [4] all the way from the RFQ (radio frequency quadrupole) injector to the end of the beam expander. Using 100000 particle simulations, very small unpredictable tails, up to 1 proton in 10000 on the edges of the roughly parabolic transverse profiles $\left(x, x^{\prime}, y, y^{\prime}\right)$, were intercepted by one of the two nonlinear focusing magnets in the beam expander. End-to-end simulations using 1000000 particles gave similar results. Thus a beam expander design using nonlinear focusing magnets was considered to be unsuitable for the APT 170-MW cw proton beam.

In order to further investigate the beam halo losses, an $800-\mathrm{MeV}$ beam expander using nonlinear focusing was designed, built, and tested [5] at the Los Alamos National Laboratory LAMPF accelerator, using a 0.1microamp average current $(80 \mathrm{~W})$ pulsed proton beam. The transverse beam profiles near the nonlinear elements in the beam expander were monitored and tuned using intercepting stepping-wire scanners, and the final $17-\mathrm{cm}$ by $170-\mathrm{cm}$ expanded beam profile was observed on a fluorescent screen. Even at this low beam current, prompt beam-loss radiation was detected near the two nonlinear octupole magnets [6] that defined the minimum nonlinear beam expander apertures. Based on these observations, the estimated beam losses with a 170-MW beam would be excessive. Another serious concern was the inability to design required suitable robust nonintercepting beam profile monitors for tuning a 170-MW beam.

The APT project beam expander baseline design was changed to a raster design [7] using linear focusing. Unlike the static DC magnetic elements in the nonlinear focusing method, the raster magnets were high-frequency AC ferrite dipole magnets. The raster magnet system required a highly redundant failsafe design and monitoring to shut off the beam in case of a component failure. The raster beam expander design used eight high-frequency raster dipole magnets, four in each plane, for redundancy. A full-scale eight-magnet raster module was built and bench-tested [8]. Unlike the nonlinear beam expander where the final beam size depends on the input beam size, the raster expander final beam size and profile depend primarily on the raster magnet current waveforms. The eight synchronized ferrite raster magnets, with $80 \mathrm{~mm}$ by $80 \mathrm{~mm}$ apertures, used triangular current waveforms at $508.441 \mathrm{~Hz}(x)$ and $573.348 \mathrm{~Hz}$ (y) (a 53/47 ratio). Each magnet was individually driven by four IGBTs (insulated gate bipolar transistor switches), and the inductive ferrite magnet energy was stored in eight separate $38 \mathrm{mF}$ capacitor banks. The peak inductive power was about $30 \mathrm{kVA}$ (100 amps, 300 volts) per magnet, and the average power loss in the IGBTs, coil, and ferrite was about $1 \mathrm{~kW}$ per magnet. The raster frequency of each magnet was derived from separate $1.024 \mathrm{MHz}$ VCXOs (voltage controlled crystal oscillators), and the eight raster magnet waveforms were synchronized in both frequency and phase to two master crystal oscillators operating at the fundamental frequencies. The magnetic field waveforms (using integration of $d B / d t$ loops in the magnet apertures) agreed very 
well with lumped-circuit computer simulations, and predicted about a $\pm 2 \%$ edge-to-edge beam uniformity of the expanded beam.

\section{ACKNOWLEDGMENTS}

This author is supported by the University of California Retirement System and the United States Social Security Administration.

[1] A. Jason and B. Blind, Confined Energy Distribution for Charged Particle Beams, U.S. Patent Number 4962317 (1990).

[2] B. Blind, Nucl. Instrum. Methods Phys. Res., Sect. B 5657, 1099 (1991).

[3] A. J. Jason and B. Blind, "Beam Expansion with Specified Final Distribution," in the 1997 Particle Accelerator Conference, Vancouver, British Columbia, Canada (1997), p. 3728, http://www.jacow.org/.
[4] T. P. Wangler et al., "New High Power Linacs and Beam Physics," in the 1997 Particle Accelerator Conference, Vancouver, British Columbia, Canada (1997), p. 915, http://www.jacow.org/.

[5] Robert E. Shafer et al. (unpublished).

[6] David Barlow et al., "Magnetic Design and Measurement of Nonlinear Multipole Magnets for the APT Beam Expander System,' in the 1997 Particle Accelerator Conference, Vancouver, British Columbia, Canada (1997), p. 3309, http://www.jacow.org/.

[7] Robert E. Shafer et al., "Overview of the APT High Energy Beam Transport and Beam Expanders," in the 1997 Particle Accelerator Conference, Vancouver, British Columbia, Canada (1997), p. 83, http://www. jacow.org/.

[8] Robert E. Shafer et al., "Testing of a Raster Magnet System for Expanding the APT Proton Beam," 1999 Particle Accelerator Conference, New York City, NY (1999), p. 3758, http://www.jacow.org/. 